Check for updates

Cite this: RSC Adv., 2018, 8, 17854

Received 1st April 2018

Accepted 8th May 2018

DOI: $10.1039 / \mathrm{c} 8 \mathrm{ra02817f}$

rsc.li/rsc-advances

\title{
Sensitive detection of free bilirubin in blood serum using $\beta$-diketone modified europium-doped yttrium oxide nanosheets as a luminescent sensor
}

\author{
Wei Yang, ${ }^{a}$ Jinfeng Xia, ${ }^{b}$ Guohong Zhou, ${ }^{b}$ Danyu Jiang ${ }^{b}$ and Qiang Li (D) *a
}

\begin{abstract}
Free bilirubin, when present in excess in the human body, can cause a multitude of diseases and disorders and even be fatal; hence, detecting it is of paramount importance. Herein, we report a luminescence quenching-based non-enzymatic method for the convenient, reliable, and rapid detection of free bilirubin in blood serum samples using sensing films (nanosheets/PS, nanosheets-tta/PS, and nanosheets-dbt/PS) as luminescent sensors. The luminescence intensity of the sensing films is linearly related to the free bilirubin concentration. Nanosheets-tta/PS demonstrated excellent sensing properties for the sensitive and reliable detection of free bilirubin in the range of $0.0-60.0 \mu \mathrm{M}$ with a correlation coefficient of 0.9915 , as compared to nanosheets/PS or nanosheets-dbt/PS. The limit of detection for the determination of free bilirubin was $41 \mathrm{nM}$. This method can be used to design a sensor-based test spot as a medical detection device for the visual detection of free bilirubin.
\end{abstract}

\section{Introduction}

Bilirubin (BR) is a yellow metabolic breakdown product of normal blood and has important biological and diagnostic significance. ${ }^{1,2}$ Free BR, also known as BR IX, has a lipophilic nature and plays a significant role in the tissue uptake and toxicity of $\mathrm{BR}^{3}{ }^{3}$ The normal concentration level of BR IX in human serum is less than $25 \mu \mathrm{mol} \mathrm{L}{ }^{-1}$, which increases to $>50$ $\mu \mathrm{mol} \mathrm{L}{ }^{-1}$ in a jaundice-infected individual. ${ }^{4-6}$ In addition to jaundice, excess BR IX can cause hepatitis, mental disorders, cerebral palsy, brain damage, and even death. ${ }^{-9}$ Therefore, precise determination of the concentration of free $\mathrm{BR}$ is extremely important.

To date, there have been many analytical methods for determining the BR IX concentration in serum samples; these include modified diazo methods, oxidation methods, bioenzymatic methods, ${ }^{10}$ separation-based methods, ${ }^{11}$ electrochemical biosensing, ${ }^{12,13}$ and fluorescence measurements. ${ }^{14}$ In most of these methods, the sample needs to be pretreated; moreover, the process of detection is complicated and indirect. The accuracy of detection using a bio-enzymatic method depends on a series of environmental conditions such as $\mathrm{pH}$ and temperature, in addition to the inconvenient processes of extraction and storage of bio-enzymes. Electrochemical sensor electrodes are easily disturbed by biological media. Therefore, there is urgent need to explore and develop a new non-

${ }^{a}$ Department of Chemistry, East China Normal University, Shanghai 200062, $P . R$. China.E-mail: qli@chem.ecnu.edu.cn

${ }^{b}$ Shanghai Institute of Ceramics, Chinese Academy of Sciences, Shanghai 200050, P. R. China enzymatic method for the direct, rapid, reliable, and visual detection of free BR in serum samples.

Fluorometric methods seem to be the most suitable means of detecting BR IX. Lanthanides have advantages such as high fluorescence quantum yields, large Stokes' shifts, strong luminescences, narrow luminescence bands, and long fluorescence lifetimes. ${ }^{15-17}$ In recent years, lanthanides have increasingly been used for biological detection. ${ }^{17,18}$

This paper describes a BR IX sensor based on Eu(III)-doped nanosheets. These nanosheets have all the advantages of Eu(III) complexes, and possess unique characteristics due to unusual structural features such as excellent two-dimensional anisotropy. More importantly, these $\mathrm{Eu}(\mathrm{III})$-doped yttrium oxide nanosheets overcome the shortcomings of $\mathrm{Eu}$ (III) complexes, and exhibit enhanced luminescence and higher stability. ${ }^{19}$ Furthermore, the above nanosheets can be used to prepare fastresponse thin-film planar optodes and optical fibers.

The Eu(III)-doped yttrium oxide nanosheet sensing films, after their successful fabrication, were modified with 2-thenoyltrifluoroacetonate (Htta) or 2-acetylbenzothiophenetrifluoroacetone (Hdbt) and coated with polystyrene (PS), in order to further improve their fluorescence intensity, monochromaticity, BR IX sensitivity, and hydrophobicity.

\section{Material and methods}

Chemicals, reagents, and apparatus

$\mathrm{Y}_{2} \mathrm{O}_{3}, \mathrm{Eu}_{2} \mathrm{O}_{3}, \mathrm{Htta}$, and Hdbt were obtained from J\&K Chemicals. BR IX was obtained from Sigma-Aldrich (USA). All chemicals and reagents in this study were of analytical grade and were used directly without further purification. Luminescence 
spectra were recorded on a FLS-980 spectrofluorometer (Edinburgh Instruments, UK) equipped with a quartz cuvette $(1.0 \mathrm{~cm}$ $\times 1.0 \mathrm{~cm}$ ) using $1 \mathrm{~nm}$-wide slits for excitation and emission. The UV-vis absorption spectra were recorded on an Agilent Cary UV8000 spectrophotometer (Tianmei, China). The photoluminescence decays of the sensing films were recorded on an FLS-980 spectrofluorometer (Edinburgh Instruments, UK) equipped with 356 and $367 \mathrm{~nm}$ lasers.

\section{Synthesis of Eu(III)-doped yttrium oxide nanosheet film}

$\mathrm{Eu}(\mathrm{III})$-doped oxide nanosheet sols were manufactured according to a procedure described previously. ${ }^{19}$ The as-received nanosheets had lateral dimensions of several hundreds of nanometers. Transmission electron microscopy (TEM) was used to confirm the ultra-thin properties of the nanosheets (Fig. 1).

The preparation process of the sensing films and detection process of BR IX are shown in Fig. 2. Firstly, the nanosheets obtained above were uniformly dispersed in $n$-butanol $(100 \mathrm{~mL})$ by $40 \mathrm{~min}$ sonication. Secondly, the positively charged nanosheets were electrophoretically deposited (EPD) onto conductive glass (FTO substrate) at $60 \mathrm{~V}$ for $10 \mathrm{~min}$, after which a uniform film was obtained. Thirdly, the film was modified with ligands, by immersing it in an ethanol solution containing Htta $(0.01 \mathrm{~g})$ or Hdbt $(0.01 \mathrm{~g})$ for $5 \mathrm{~min}$, then dried in air. The above coating process was repeated thrice to obtain a luminescent film of Htta/Hdbt-modified nanosheets. Finally, the BR sensing film was obtained by dip-coating it in dry $\mathrm{CH}_{2} \mathrm{Cl}_{2}(10 \mathrm{~mL})$ containing PS $(0.1 \mathrm{~g})$ at a rate of $2500 \mu \mathrm{m} \mathrm{s}^{-1}$.

Fresh human blood samples were collected from healthy volunteers. All experiments were performed in accordance with the Guidelines "Declaration of Helsinki (2002 edition)" and "Measures for Ethical Review of Biomedical Research involving People", and experiment approved by "the Academic Ethics Committee of East China Normal university". Informed consents were obtained from human participants of this study. The original content of BR IX in these samples was removed using a reported method. ${ }^{20-22}$ Serum samples containing BR IX $(1-200 \mu \mathrm{M})$ were equilibrated at room temperature (RT). Thereafter, $30 \mu \mathrm{L}$ of the test sample was dropped onto the

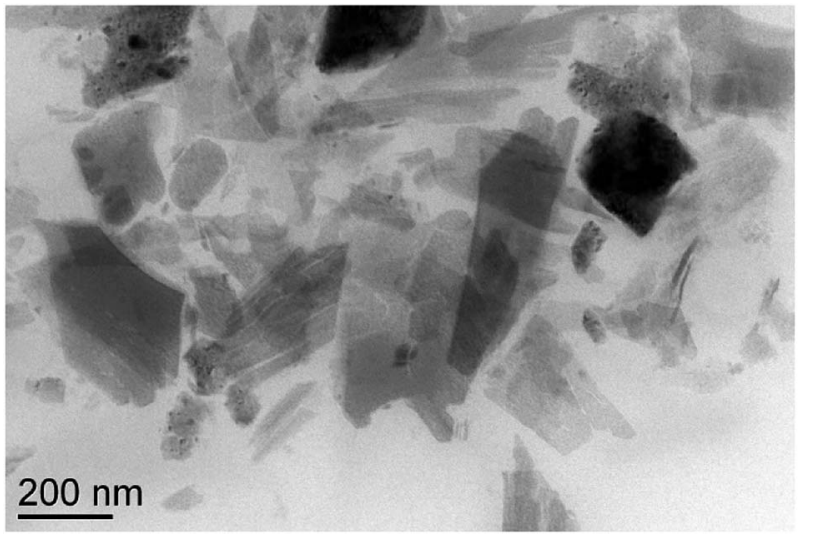

Fig. 1 Transmission electron microscopy (TEM) image of Eu(III)-doped oxide nanosheets.

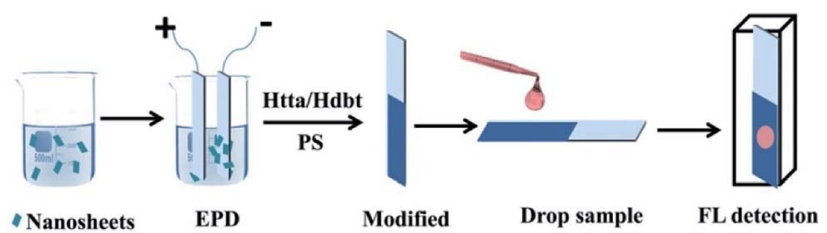

Fig. 2 Processes of fabrication and sample detection using the nanosheets-based sensing film.

surface of the sensing films, as shown in Fig. 2. The sensing film was placed in a cuvette and the BR IX concentration was detected by a fluorescence spectrophotometer. In another method, the sensing film was placed directly under UV light to evaluate its efficiency as a point-of-care device for visually detecting BR IX.

\section{Detection of BR IX by three sensing films}

Fig. 3 shows the excitation spectra of the nanosheets without and with the ligands (Htta or Hdbt), which were recorded at 274, 356, and $367 \mathrm{~nm}$, for the PS-encapsulated Eu(III)-doped yttrium oxide nanosheets (nanosheets/PS), PS-encapsulated Httamodified $\mathrm{Eu}(\mathrm{III})$-doped yttrium oxide nanosheets, (nanosheetstta/PS), and PS-encapsulated Hdbt-modified Eu(III)-doped yttrium oxide nanosheets (nanosheets-dbt/PS), respectively. The luminescence responses toward different concentrations of BR IX were determined in triplicate to provide average values.

\section{Results and discussion}

\section{Luminescence response of sensing films to BR IX}

As shown in Fig. 4, nanosheets/PS, nanosheets-tta/PS, and nanosheets-dbt/PS have the same emission maxima at $614 \mathrm{~nm}$, which is the characteristic emission from the ${ }^{5} \mathrm{D}_{0}{ }^{-}{ }^{7} \mathrm{~F}_{2}$ transition of Eu(III). Nanosheets/PS, nanosheets-tta/PS, and nanosheets$\mathrm{dbt} / \mathrm{PS}$ were excited at 274, 356, and $367 \mathrm{~nm}$, respectively, and the emission peaks were monitored in the $550-750 \mathrm{~nm}$ range. As shown in Fig. 4(b) and (c), the luminescence intensity of

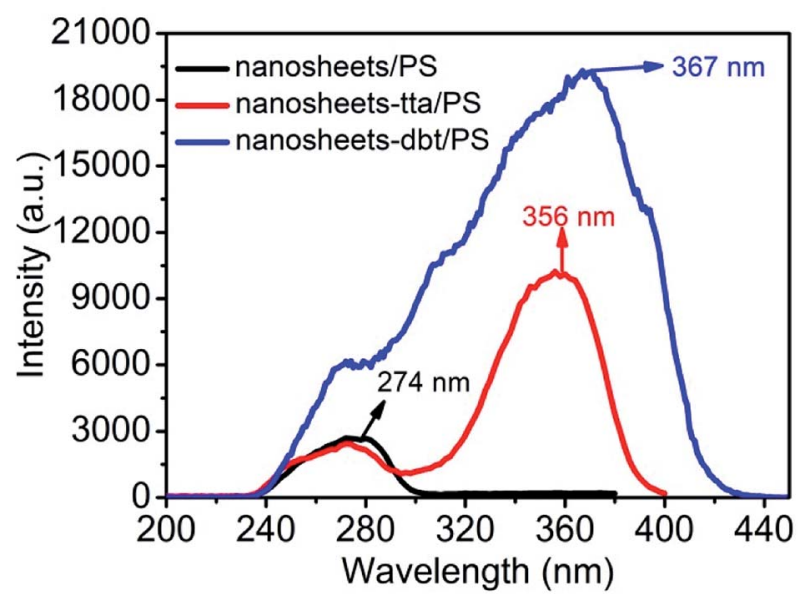

Fig. 3 Excitation of nanosheets/PS, nanosheets-tta/PS, and nanosheets-dbt/PS 

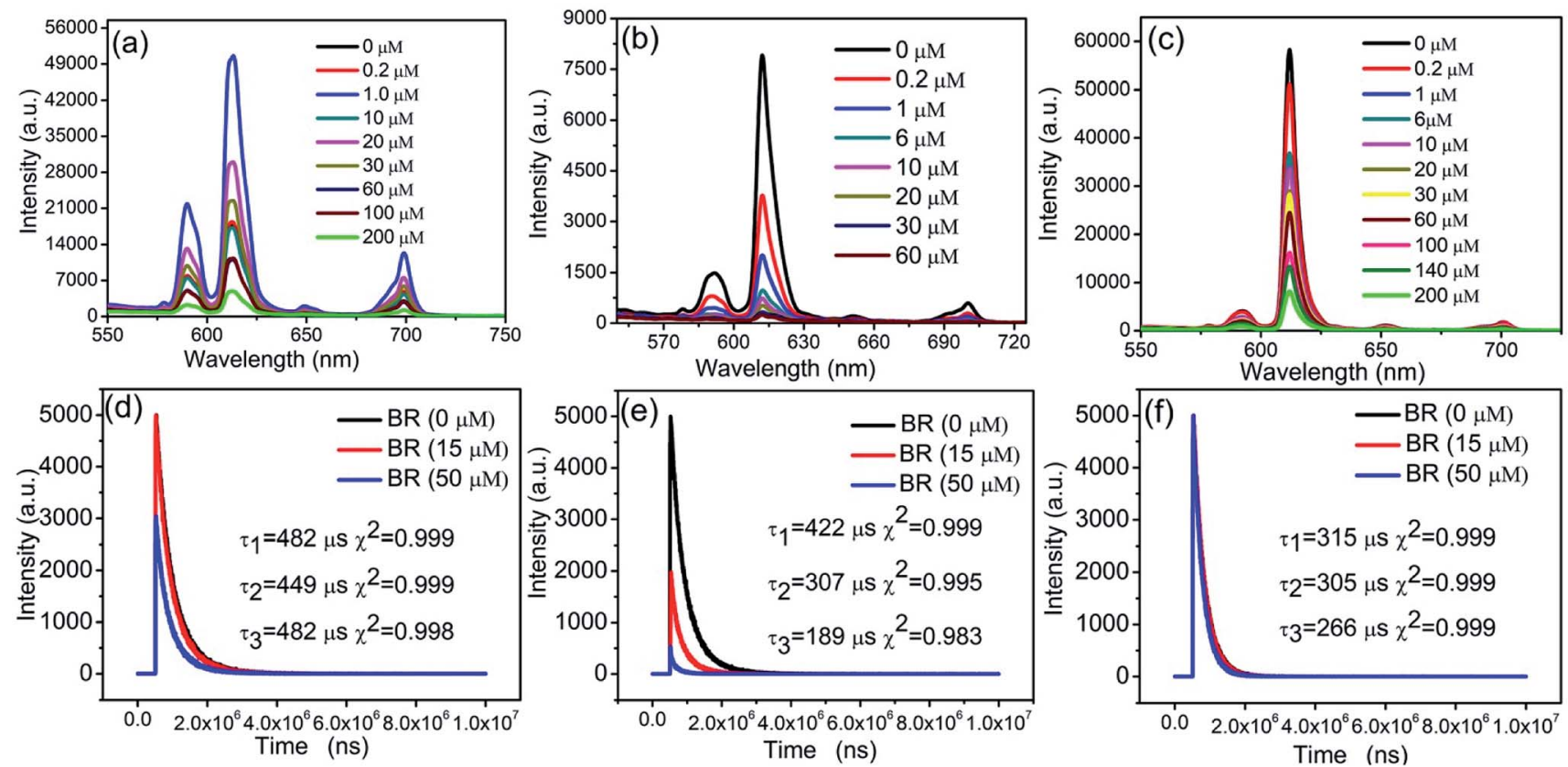

Fig. 4 Luminescence emission spectra of nanosheets/PS (a), nanosheets-tta/PS (b), and nanosheets-dbt/PS (c). Fluorescence lifetime of nanosheets/PS (d), nanosheets-tta/PS (e), and nanosheets-dbt/PS (f).

nanosheets-tta/PS and nanosheets-dbt/PS gradually decreases with increasing BR IX concentration. Only when the BR IX concentration is greater than $20 \mu \mathrm{M}$, does the luminescence intensity of nanosheets/PS decrease with increasing BR IX concentration, which is not suitable for detecting the BR IX concentration in the normal range $(6.0-17.1 \mu \mathrm{M})$. The detection ranges of nanosheets-tta/PS $(0.0-60 \mu \mathrm{M})$ and nanosheets-dbt/PS $(0-200 \mu \mathrm{M})$ observed herein are suitable to detect the BR IX concentration in human serum samples. The luminescence intensity of nanosheets-tta/PS is almost completely quenched at a BR IX concentration of $60 \mu \mathrm{M}$, (Fig. 4(b)), whereas the nanosheets-dbt/PS retains a strong luminescence intensity even at $200 \mu \mathrm{M}$ (Fig. 4(c)). An investigation of the effect of BR IX on the fluorescence lifetime of the three sensing films in Fig. 4(d)(f) revealed that BR IX exhibited no regularity in the fluorescence lifetime of nanosheets/PS, with $15 \mu \mathrm{M}$ of BR IX having a significant influence, and $50 \mu \mathrm{M}$, no influence. The properties of the sensing film containing nanosheets-tta/PS were greatly affected by the BR IX concentration; its fluorescence lifetime decreased with increasing BR IX concentration $(0-50 \mu \mathrm{m})$. The fluorescence lifetime decayed from $422 \mu \mathrm{s}$ to $189 \mu \mathrm{s}$, with a reasonably high decay efficiency, which further demonstrates that the highly sensitive nanosheets-tta/PS can be used for the detection of BR IX. The fluorescence lifetime of nanosheets-dbt/ PS also decreased with the increase in BR IX concentration; its attenuation was greater than nanosheets/PS and less than nanosheets-tta/PS.

\section{Interactions of BR IX with sensing films}

There are two possible interactions between BR IX and nanosheets or BR IX and the ligands (Htta, Hdbt). The UV-vis absorption spectra of BR IX, nanosheets, Htta, and Hdbt are shown in Fig. 5. It can be seen that a narrow absorption peak of rare earth ion $(\operatorname{Re}(\mathrm{III}))$ appears at $238 \mathrm{~nm}$ in Fig. 5(a), which is contributed to the $\mathrm{Y}^{3+}-\mathrm{O}^{2-}$ charge transfer. ${ }^{23}$ The band at
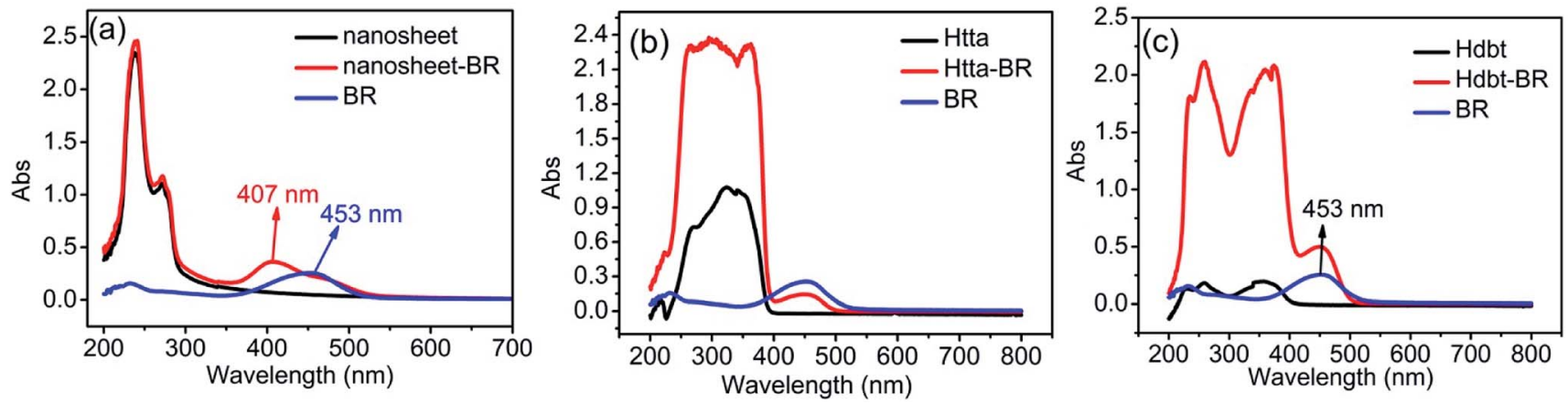

Fig. 5 Absorption spectra of nanosheets, nanosheets-BR IX, BR IX (a); Htta, Htta-BR IX, BR IX (b); Hdbt, Hdbt-BR IX, BR IX (c). 

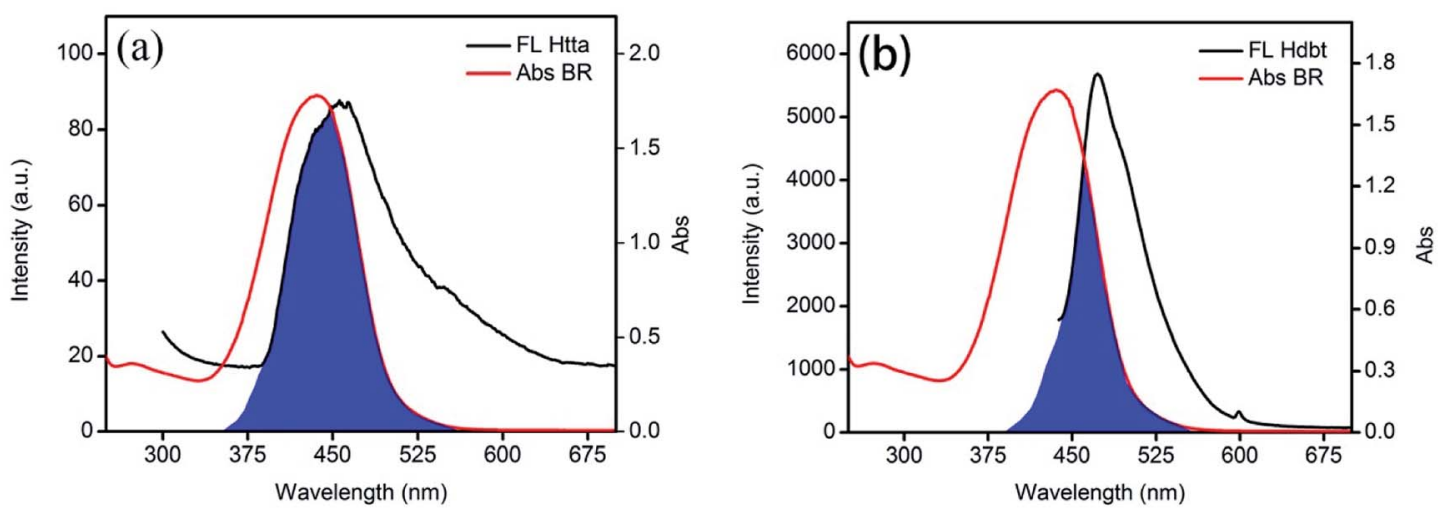

Fig. 6 Overlap of the fluorescence emission spectra of Htta (a) and Hdbt (b) with the absorption spectra of BR IX.

$271 \mathrm{~nm}$ in Fig. 5(a) was attributed to the $\mathrm{Eu}^{3+}-\mathrm{O}^{2-}$ charge transfer, ${ }^{24}$ The bands appearing in the ligands at $200-300 \mathrm{~nm}$ in Fig. 5(b) and (c) are due to $\mathrm{n} \rightarrow \pi^{*}$ transition of keto-enol tautomerism. ${ }^{25}$ The broad bands in the $300-400 \mathrm{~nm}$ range for the ligands are due to the singlet-singlet $\pi \rightarrow \pi^{*}$ transition of enol absorptions. ${ }^{26,27}$ The maximum-absorption band for BR IX at around $450 \mathrm{~nm}$ is due to the superposition of two electronic transitions in BR IX, near $480 \mathrm{~nm}$ and around $430 \mathrm{~nm} .{ }^{28}$ The absorption spectra of BR IX shift from $453 \mathrm{~nm}$ to $407 \mathrm{~nm}$ after adding the nanosheets, which reveals that BR IX coordinates to $\operatorname{Re}(\mathrm{III})$ in the nanosheets, as reported in literature. ${ }^{29,30}$ Comparison of the absorbance spectra of BR IX before and after the addition of ligands (Htta, Hdbt) reveals no shift and no new peak generation. Therefore, BR IX does not coordinate with Htta and Hdbt.

Although BR IX and Re can be coordinated, BR IX can be better detected by nanosheets-tta/PS and nanosheets-dbt/PS than nanosheet/PS. As seen in Fig. 6, the emission spectra of Htta (456 $\mathrm{nm}$ ) and Hdbt (473 nm) overlap with the UV absorption spectrum (453 nm) of BR IX. The overlapping part is marked in blue; it is clear that the overlapping area of Htta and BR IX is larger than that of Hdbt and BR IX. The excitation peak of the nanosheets is located at $274 \mathrm{~nm}$, which has almost no overlap with the excitation peak of BR IX. According to Föster's resonance energy transfer theory (FRET), ${ }^{31,32}$ the larger the overlap area, the better the energy matching, and the more energy the ligands deliver to BR IX. Therefore, Htta can deliver energy to BR IX more efficiently than Hdbt and nanosheets. This result is also consistent with the fluorescence lifetime results in Fig. 4.

The luminescence mechanism of the BR IX-quenching nanosheets-tta/PS and nanosheets-dbt/PS is due to the antenna effect and Föster's nonradiative energy transfer theory. The ligands (Htta, Hdbt) on nanosheets-tta/PS and nanosheets$\mathrm{dbt} / \mathrm{PS}$ are coordinated with $\mathrm{Eu}(\mathrm{III})$, respectively, and the absorbed energy is transferred from the ligands to the luminescent center. The characteristic emissions of $\mathrm{Eu}(\mathrm{III})$ then appear. After dropping BR IX on the sensing films, BR IX is coordinated with Re in the nanosheets, and the energy transferred by the ligand matches that absorbed by BR IX. Consequently, the energy delivered by the ligands to the luminescent center is reduced, due to which the luminescence intensity of $\mathrm{Eu}$ (III) also decreases. The quenching process can be expressed by the following equation: $[\mathrm{C}]+n[\mathrm{Q}] \rightarrow\left[\mathrm{C}^{\cdots} n \mathrm{Q}\right]^{33,34}(\mathrm{C}=$ nanosheets-tta/PS, or nanosheets-dbt/PS).

The energy was transferred from coordinated ligands to $n$ equivalents of coordinated BR IX, and BR IX concentration is expressed as [Q]. The luminescence of nanosheets-tta/PS and nanosheets-dbt/PS are quenched as described above. The regression lines of nanosheets-tta/PS and nanosheets-dbt/PS were plotted using the following equations: ${ }^{20}$
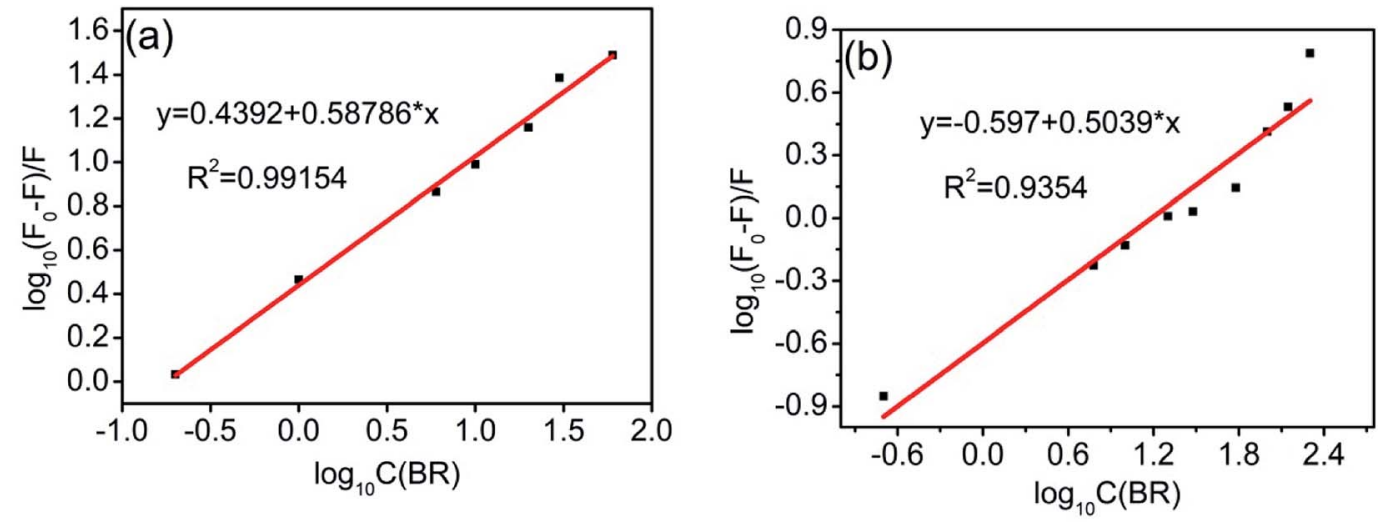

Fig. 7 Plot of $\log \left[\left(F_{0}-F\right) / F\right]$ as a function of $\log [B R I X]$ nanosheets-tta/PS (a), and nanosheets-dbt/PS (b). 
Table 1 Methods for the determination of BR IX

\begin{tabular}{|c|c|c|c|c|c|}
\hline Methods & $\begin{array}{l}\text { Linear range } \\
\left(\mu \mathrm{mol} \mathrm{L}{ }^{-1}\right)\end{array}$ & $\begin{array}{l}\text { Detection limit } \\
\left(\mathrm{nmol} \mathrm{L}^{-1}\right)\end{array}$ & Principal part & $\begin{array}{l}\text { Correlation } \\
\text { coefficient }\end{array}$ & \\
\hline Molecular imprinting & $1.71-85.51$ & 770 & (PHEMATrp) nanofilm (MIP) & $R^{2}=0.98$ & 36 \\
\hline Fluorescence-based method & $33-300$ & 52 & $\mathrm{Ru}(\text { bipy })_{3}{ }^{2+}$ & $R^{2}=0.998$ & 37 \\
\hline Fluorescence-based method & $0.0001-100$ & $5.9 \times 10^{-3}$ & MOF & $R^{2}=0.998$ & 3 \\
\hline Photoelectrochemical & $0.03-28$ & 1 & $\mathrm{TiO}_{2}$-polypyrrole & $R^{2}=0.998$ & 39 \\
\hline Fluorescent-based method & $0.0002-0.002$ & 0.12 & S,N-doped carbon dots & $R^{2}=0.98$ & 14 \\
\hline The developed method & $0-60$ & 41 & Nanosheets-tta/PS & $R^{2}=0.991$ & - \\
\hline
\end{tabular}

$$
\begin{gathered}
K_{\mathrm{A}}=\frac{[\mathrm{C} \cdots n \mathrm{Q}]}{[\mathrm{C}][\mathrm{Q}]^{n}}=\frac{\left[\mathrm{C}_{0}\right]-[\mathrm{C}]}{[\mathrm{C}][\mathrm{Q}]^{n}} \\
\frac{[\mathrm{C}]}{\left[\mathrm{C}_{0}\right]}=\frac{F}{F_{0}} \\
K_{\mathrm{A}}[\mathrm{Q}]^{n}=\frac{F_{0}-F}{F} \\
\log \frac{F_{0}-F}{F}=\log K_{\mathrm{A}}+n \log [\mathrm{Q}]
\end{gathered}
$$

where $\mathrm{C}$ and $\mathrm{C}_{0}$ are the sensing films with and without BR IX; $F$ and $F_{0}$ are the luminescence intensities of the sensing films with and without BR IX, respectively.

Eqn (4) was used to generate luminescence response curves for the detection of BR IX by the sensing films. As shown in Fig. 7, the logarithm of the luminescence intensity of nanosheets-tta/PS and nanosheets-dbt/PS $\left(\log \left(\left(F_{0}-F\right) / F\right)\right)$ is proportional to the logarithm of the BR IX concentration (log[BR IX]), in the concentration range of $1-60 \mu \mathrm{M}$ and 1-200 $\mu \mathrm{M}$, respectively. The detection limit $\left(C_{\mathrm{LOD}}\right)$ is defined by IUPAC and calculated by the formula $C_{\mathrm{LOD}}=3 S_{\mathrm{b}} / m^{35,36}$ The limits of detection of nanosheetstta/PS and nanosheets-dbt/PS were $41 \mathrm{nM}$ and $138 \mathrm{nM}$, respectively. Compared to nanosheets-dbt/PS, nanosheets-tta/PS exhibits better linearity $\left(R^{2}=0.99154\right)$ and a lower detection

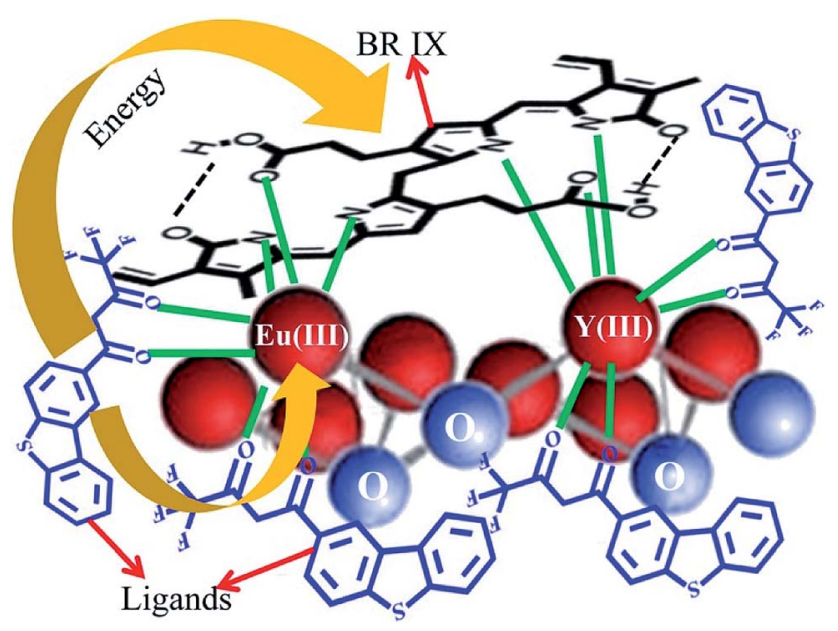

Fig. 8 Energy transfer mechanism for luminescence quenching in BR IX-nanosheet-tta/PS and BR IX-nanosheets-dbt/PS. limit. The broad detection range, high detection reliability, and ultra-low detection limit of nanosheets-tta/PS demonstrates its potential use as an excellent visualization sensor of BR IX.

The various methods used for detecting BR IX are listed in Table 1. The molecular imprinting-based method has a wide detection range, but a very high minimum detection limit. The $\mathrm{Ru}$ (bipy) ${ }_{3}{ }^{2+}$-based fluorescence method ${ }^{37}$ has a sufficiently low detection limit; however, its detection range $(33-300 \mu \mathrm{M})$ is unsuitable for detecting BR IX, because the normal human bilirubin concentration is lower than $25 \mu \mathrm{M}$. The fluorescent protein-based method ${ }^{38}$ and (BOx)-based method ${ }^{7}$ both seem to be the best candidates for BR IX testing; however, they require fluorescent proteins or enzymes, which pose a significant challenge for probe preparation and maintenance. The photoelectrochemical method ${ }^{39}$ has a low detection limit, but a very narrow detection range. The Multiple Organ Failure (MOF)based fluorescence method $^{3}$ has a wide detection range and a low detection limit. However, the fluorescence intensity of MOF is small, and the preparation of MOF is complicated. Electrochemical biosensing-based method $^{13}$ has a high sensitivity and low detection limit. The bioelectrode was successfully applied to measure the bilirubin content in spiked serum samples. Among the methods listed in Table 1, only the S,Ndoped carbon dots-based method ${ }^{\mathbf{1 4}}$ and the method developed in this study can be used to prepare a solid-state sensor. The sensor made from S,N-doped carbon dots has a very high sensitivity; however, its detection range is not suitable to detect BR IX in humans. Nanosheets-tta/PS as a solid-state sensor not only has a low detection limit, but also has a suitable detection range for BR IX detection in humans. It can be employed as a visual inspection instrument in the future.

The number of BR IX molecules coordinated with Eu(III) has a significant influence on the energy transfer efficiency. The number of BR IX molecules $(n)$ coordinated with $\mathrm{Eu}$ (III) onnanosheet-tta/PS and nanosheet-dbt/PS $(n)$ is obtained from the slope of their fitted line according to eqn (4), and is 0.58 and 0.50 , respectively. The UV absorption spectra in Fig. 5 reveal that the BR IX molecule is coordinated with $\mathrm{Eu}(\mathrm{III}) / \mathrm{Y}(\mathrm{III})$. As shown in Fig. 8, the middle club model is the structure of the nanosheet, the black molecular is the molecule of BR IX, and the blue molecules refer to ligands (Htta or Hdbt). One BR IX was coordinated to two Re(III), one Eu(III) and one $\mathrm{Y}(\mathrm{III})$, and there are multiple ligands coordinated with each $\mathrm{Eu}(\mathrm{III}) / \mathrm{Y}(\mathrm{III})$. 
When the ligands transfer energy to the luminescent center, the energy transferred by the ligand is absorbed by BR IX, because the energy of the ligand matches that absorbed by BR IX, resulting in the net reduction of the energy obtained from the luminescent center, and thereby causing luminescence quenching.

\section{Conclusions}

We successfully prepared an $\mathrm{Eu}(\mathrm{III})$-doped yttrium oxide nanosheet film and modified it with $\mathrm{Htta} / \mathrm{Hdbt}$ and PS to improve the luminescence and service life of the film. The obtained sensing film, nanosheet-tta/PS, could sensitively and reliably detect BR IX in human serum samples. The luminescence intensity of nanosheet-tta/PS was effectively quenched by BR IX via a FRET process. Since the rare-earth nanosheet can be deposited by electrophoretic deposition or spin coating, it can be coated on a conductive material or fiber surface to enable the easier detection of BR IX by the sensing film. Nanosheet-tta/PS can thus be used as a film-based sensor in testing for BR IX.

\section{Conflicts of interest}

There are no conflicts to declare.

\section{References}

1 J. Gao, W. Xu, K. Han, L. Zhu, L. Gao and X. Shang, Neuropsychiatr. Dis. Treat., 2018, 14, 83-93.

2 C.-F. Ma, Q. Gao, J. Zhou, Q.-X. Chen, B. Han, K.-S. Xia and C.-G. Zhou, RSC Adv., 2017, 7, 2081-2091.

3 Y. Du, X. Li, X. Lv and Q. Jia, ACS Appl. Mater. Interfaces, 2017, 9, 30925-30932.

4 M. F. Xue and H. P. Hospital, Clinical Research \& Practice, 2017.

5 Q. Feng, Y. Du, C. Zhang, Z. Zheng, F. Hu, Z. Wang and C. Wang, Sens. Actuators, B, 2013, 185, 337-344.

6 S. K. Chou and M. J. Syu, Biomaterials, 2009, 30, 1255-1262.

7 V. Hooda, A. Gahlaut, A. Gothwal and V. Hooda, Biotechnol. Lett., 2017, 39, 1-10.

8 J. Wang, X. Wu, Y. Li, X. Han, H. Hu, F. Wang, C. Yu, X. Li, K. Yang and J. Yuan, Acta Diabetol., 2016, 54, 1-8.

9 T. Balamurugan and S. Berchmans, RSC Adv., 2015, 5, 5047050477.

10 M. E. Khlupova, I. S. Vasil'Eva, G. P. Shumakovich, O. V. Morozova, V. A. Chertkov, A. K. Shestakova, A. V. Kisin and A. I. Yaropolov, Biochemistry, 2015, 80, 233241.

11 E. Salimi, A. Ghaee and A. F. Ismail, Mater. Lett., 2018, 215, 276-279.

12 T. Ikeda, H. Tatsumi, H. Katano, M. Wanibuchi, T. Hibi and T. Kajino, Anal. Sci., 2008, 24, 237-241.

13 M. Santhosh, S. R. Chinnadayyala, N. K. Singh and P. Goswami, Bioelectrochemistry, 2016, 111, 7-14.

14 R. R. Anjana, J. S. A. Devi, M. Jayasree, R. S. Aparna, B. Aswathy, G. L. Praveen, G. M. Lekha and G. Sony, Microchim. Acta, 2018, 185, 11-21.
15 P. Cortelletti, A. Skripka, C. Facciotti, M. Pedroni, G. Caputo, N. Pinna, M. Quintanilla, A. Benayas, F. Vetrone and A. Speghini, Nanoscale, 2017, 10, 2568-2576.

16 F. Wu, M. Mao, Y. Cen, H. Yang, Z. Qin and L. Ma, RSC Adv., 2017, 7, 12217-12223.

17 P. J. Cywiński, M. Pietraszkiewicz, M. Maciejczyk, K. Górski, T. Hammann, K. Liermann, B.-R. Paulke and H.-G. Löhmannsröben, RSC Adv., 2016, 6, 115068-115073.

18 B. B. C. Holanda, R. B. Guerra, A. O. Legendre, D. F. Almeida, T. F. C. Fraga-Silva, Â. C. Finato, J. Venturini and G. Bannach, Thermochim. Acta, 2016, 647.

19 L. Zhang, D. Jiang, J. Xia, C. Li, N. Zhang and Q. Li, RSC Adv., 2014, 4, 17648.

20 M. Santhosh, S. R. Chinnadayyala, A. Kakoti and P. Goswami, Biosens. Bioelectron., 2014, 59, 370-376.

21 A. H. Huber, B. Zhu, T. Kwan, J. P. Kampf, T. Hegyi and A. M. Kleinfeld, Clin. Chem., 2012, 58, 869-876.

22 M. Martelanc, L. Ziberna, S. Passamonti and M. Franko, Anal. Chim. Acta, 2014, 809, 174-182.

23 Q. Dai, M. E. Foley, C. J. Breshike, A. Lita and G. F. Strouse, J. Am. Chem. Soc., 2011, 133, 15475-15486.

24 Y. Sohn, Ceram. Int., 2014, 40, 2467-2475.

25 X. Zhang, Z. C. Li, C. F. Lao, D. C. Zou, F. Z. Lu, G. Q. Chen, F. S. Du and F. M. Li, Polymer, 2006, 47, 3390-3400.

26 S. G. Liu, M. L. Gong, S. Wang and X. M. Tan, Spectrochim. Acta, Part A, 2009, 74, 731-734.

27 S. G. Liu, R. K. Pan, X. P. Zhou, X. L. Wen, Y. Z. Chen, S. Wang and X. B. Shi, Inorg. Chim. Acta, 2013, 395, 119-123.

28 P. Novotna, F. Kralik and M. Urbanova, Biophys. Chem., 2015, 205, 41-50.

29 I. Spasojević, I. Batinić-Haberle, R. D. Stevens, P. Hambright, A. N. Thorpe, J. Grodkowski, P. Neta and I. Fridovich, Inorg. Chem., 2001, 40, 726-739.

30 Q. K. Zhuang, H. C. Dai and H. Liu, Electroanalysis, 2015, 11, 1368-1371.

31 S. Roy and T. K. Das, Nanosci. Nanotechnol. Lett., 2014, 16, 5434-5437.

32 M. S. Smirnov, Opt. Spectrosc., 2017, 123, 705-716.

33 E. N. D. Silva, P. A. B. D. Silva, A. E. Graminha, P. F. D. Oliveira, J. L. Damasceno, D. C. Tavares, A. A. Batista and G. V. Poelhsitz, Bioinorg. Chem. Appl., 2017, 2017, 1-9.

34 F. Fang, D. Q. Pan, M. J. Qiu, T. T. Liu, M. Jiang, Q. Wang and J. H. Shi, Luminescence, 2016, 31, 1242-1250.

35 T. P. Robinson, G. W. Wardell-Johnson, G. Pracilio, C. Brown, R. Corner and R. D. V. Klinken, International Journal of Applied Earth Observation \& Geoinformation, 2016, 44, 23-30.

36 Ç. Çiçek, F. Yılmaz, E. Özgür, H. Yavuz and A. Denizli, Chemosensors, 2016, 4, 21-32.

37 S. M. Wabaidur, G. E. Eldesoky and Z. A. Alothman, Luminescence, 2018, 33, 625-629.

38 S. Iwatani, H. Nakamura, D. Kurokawa, K. Yamana, K. Nishida, S. Fukushima, T. Koda, N. Nishimura, H. Nishio and K. Iijima, Sci. Rep., 2016, 6, 28489-28496.

39 C. Zhang, W. Bai and Z. Yang, Electrochim. Acta, 2016, 187, 451-456. 